\title{
A hybrid on-line ECG segmenting system for long-term monitoring
}

\author{
P. Várady ${ }^{1}$, Z. Benyó ${ }^{1}$, T. Micsik ${ }^{1}$, Gy. Moser $^{2}$ \\ ${ }^{1}$ Department of Control Engineering and Information Technology (IIT), \\ Budapest University of Technology and Economics (BUTE) and \\ ${ }^{2}$ Coronary Care Unit of the Szt. Rókus Hospital, Budapest, Hungary
}

Received: June 14, 2000

Accepted: October 23, 2000

\begin{abstract}
This paper introduces a new hybrid ECG beat segmenting system, which can be applied in the processing unit of single-channel, long-term ECG monitors for the on-line segmentation of the ECG signal. Numerous ECG segmentation techniques are already existing and applied, however sufficiently robust and reliable methods currently require more than one ECG signal channel and quite complex computations, which are practically not feasible in stand-alone, lowcost monitors. Our new system approach presents a time domain segmentation technique based on a priori physiological and morphological information of the ECG beat. The segmentation is carried out after classifying the ECG beat, using the linear approximation of the filtered ECG signal and considering the pathophysiological properties as well. The proposed algorithms require moderate computational power, allowing the practical realization in battery powered stand-alone long-term cardiac monitors or small-sized cardiac defibrillators. The prototype version of the system was implemented in Matlab. The test and evaluation of the system was carried out with the help of reference signal databases.
\end{abstract}

Keywords: ECG segmentation, ECG signal processing, linear approximation, neural networks, wavelet transformation

\section{Importance of ECG and its segments}

The first electrocardiogram (ECG) was recorded in 1887, but it wasn't until the early 1900s', when they realized its diagnostic power. Einthoven gave names to the waves and segments seen on the record and standardized the leads we have been using

Correspondence should be addressed to

Péter Várady

Department of Control Engineering and Information Technology (IIT),

Budapest University of Technology and Economics

H-1117 Budapest, Pázmány sétány 1/d, Hungary

E-mail: varady@iit.bme.hu 
since then (Fig. 1). The knowledge on electrocardiography has been getting even deeper, as well its instrumental backup. For today there are newer recording techniques available: vectorcardiography, transoesphageal leads, surface mapping, intracardial leads etc. The largest and newest foresteps are taken in therapy, as well, as you can cure malignant arrhythmias eliminating their pathomorphological substrate (1).

The problem of ECG segmentation is to detect the correct position of the individual ECG characteristic points, to find the ECG-waves (determine their timedomain features: length, height and area) and to calculate various time intervals between these characteristic points (Fig. 1).

If you have an ECG record in your hand, you must judge it to reveal information about the heart (2). The summary must consist of the place the beat is set out and the activation sequence. If there is a $P$-wave, its shape and axis could tell us a lot about the electrical activity of the atrias, and through this we can gain a lot about the anatomical structure and metabolism in it. Since the sinus node, the conductor of the heart which determines the frequency of the heart is placed in the right atrium not far from the cava vessels, studying $\mathrm{P}$-waves plays an important role in diagnosing arrhythmias and conduction disorders.

The activation of the atrias is a bit delayed before continuing into the ventricles, this is characterized by $P Q$-time. Looking thoroughly at this part of the ECG record we can draw conclusions about the state of the heart's conduction system and about certain circumstances that could make effects on it: metabolic disorders, intracardial and extracardial causes, activity of neural system etc.

The very next and most interesting part of the record is the QRS-complex that delivers very important information. We can determine and follow the sequence and process how the heart becomes activated, assume the conditions or even the disorders of myocardium. We have to underline that we cannot judge from only a part of an ECGrecord, we have to have a look at the whole of it in its totality.

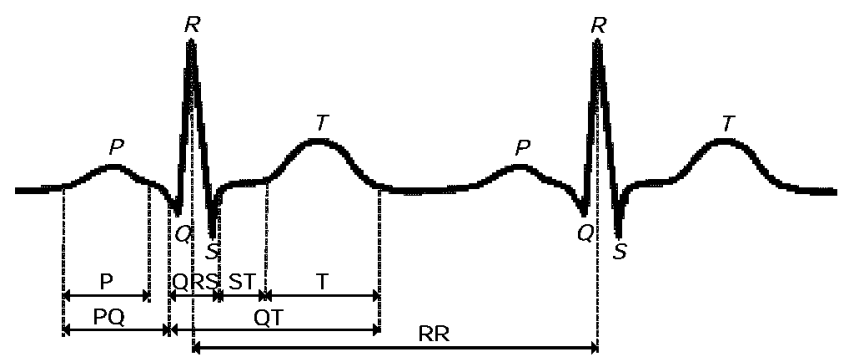

Fig. 1. Characteristic points and time intervals on normal ECG 
And there is another important point that you have to compare ECG-record with the clinical findings. We can come to incorrect conclusions because for example any changes in the QRS-complex could result in T-wave changing, which you could take for a sign of ischaemia if you do not take a look at the preceded QRS-complex. Cardiologists prefer examining the ECG graphs with their own eyes to taking the diagnoses provided by machines for granted.

The $S T$ part preceded by QRS complex characterizes the repolarisation. The ST$\mathrm{T}$ is influenced by the actual physical state of the patient in many ways such as intraventricular conduction disorder, metabolism, ischaemia etc. If the patient is suffering from any kind of heart diseases, it results in the declination of the process and way of depolarisation and repolarisation as compared to the normal one. In case of ischemic heart disease the first warning signs are usually visible here. We have to examine the characteristic features, the position, form, quantity, axis etc. of the ST-T waves and parts in all the leads.

During the analysis we cannot forget about the $Q T$ time that is influenced by the condition of the myocardium, by deviations of metabolism and conduction disorders. The QT section characterizes the ventricle revolution. Although its length can vary, still there is an average corresponding time limit depending on heart rate, that should be kept in adherence.

Short and long haul observing of the changes in $R R$-distances provides us an insight to the function of the autonomic nervous system. We get the characteristics of heart rate variability (HRV) by analyzing and charting the distance between RR heights, and from the HRV we can predict the vagosympathetical distony. If the sympathetic overweight does not let the vagal effect come alive, it leads to catecholaminaemy and to more extensive cardiovascular risk.

Comparison of ECG records of the same patient recorded at different times and under different clinical conditions reveals further important pieces of information.

\section{State of the art ECG analysis}

Nowadays ECG-machines do not only print out the curve, but also give the analysis of it. It has the advantage to call our attention to several shady aspects, but has the disadvantage that these machines are more expensive ones and need computers with better capacity in order to remain quick enough. This is why it is not always available at consulting rooms and sometimes even the lack of expertise make the proper usage difficult or impossible. The final output of the machines can also contain slight mistakes. Yet it is important to highlight that the different waves, time periods of certain parts and other features of the ECG-records depend on plenty of factors and 
should be treated accordingly. To do an analysis accurate like this a computer that has the adequate amount of programs, the ability to count properly, the reliability high level and accuracy is essential.

With the help of long-term (Holter) monitoring the possibilities have become much better in diagnosing myocardial ischaemia and malignant arrhythmias. From the 24-hours-long records we can get some answer to the frequency, seriousness and the stimuli of ischemic and arrhythmic periods. Although, Holter-monitor pinpoints those pathologic waves and periods that are previously determined by the investigator out of several thousands of recorded beats, it is not able to diagnose other unhealthy beats.

There are so-called event monitors existing that are applied and turned on by the patients during complaints. These can be used even for weeks if necessary. By now Holter-monitoring have already become a frequently used assistance in hands of cardiology.

Electronic activity of the more sophisticated pacemakers (pacemakercardioverter-defibrillator) is depending on their built-in arrhythmia recognising program that recognises the function errors that are determined before. Considering the fact that these are implants, their size and weight plays very important role.

The thorough introduction of existing systems and methods is far beyond the scope of this paper. We just highlight here some ideas, which led us at the beginning of this study:

- the total automatic computer analysis of ECG records even today is highly impossible and unsolved, due to the frequent and widely variable arrhythmia forms, especially in case of restricted number of ECG leads,

- the analyser programs work on a beat-by-beat basis, which means that at first they look for the R-peaks, then in knowledge of the R-peak's features, they continues on examining the other parts of the record. In case of arrhythmias the frequency of abnormal beats is high and many algorithms are not prepared enough for these irregularities, which can result in errors,

- the correct segmentation of the individual beats is not enough robustly supported, especially in case of single-channel ECG and on-line processing.

ECG segmentation has a long history in ECG signal processing. There are various kinds of algorithms constructed and proposed for ECG segmentation. The most robust methods use multi-channel ECG records, deriving the required parameters from various ECG leads by the means of complex and time-consuming computations $(3,4,5)$. Some approaches were proposed for the single-channel segmentation, however without the required robustness and support of complex cardiac arrhythmias other than normal sinus rhythms $(6,8)$. The methods (3-6) work in the time domain, either using a 
morphological or a linear approximated analysis of the ECG. The frequency domain segmentation is practically not possible, due to the wide variations and overlaps between the spectra of the ECG waves and in case of the spectral content of normal and aberrated beat segments. Spectral methods can be used for the classification of various type of ECG beats (10). The time-frequency (mainly wavelet transformation based) analysis is another promising technique used in this field, however its practical applicability in stand-alone, on-line monitors is limited, due to the expensive computations (the Wavelet transforms should be calculated at 3-5 different scales) (7-9).

In our study we would like to propose a new hybrid method, combining the advantages of several well-known ECG signal-processing techniques. We designed and implemented a system, whereby the following requirements were considered:

- one single ECG signal channel,

- on-line (beat-by-beat) segmentation,

- robust segmentation of various type of beats,

- use of physiological background knowledge,

- robustness against various noises,

- inexpensive computations,

- modular and expandable structure.

\section{Basic concept}

Due to the complex morphology of ECG waveforms, the obtaining of the exact location of characteristic points requires a sophisticated method, which should be based on the a priori information on the beat type and physiological knowledge on the properties of ECG waves (2). This approach is very similar to that human cardiologists are using. Various type of beats can have very different waveforms, containing even not all characteristic points in the beat. On the other hand, the variation of the same type of beat's shape in a patient and among various patients makes the problem even more complicated. These considerations underline the importance of a priori information on the beat type. Without this, it would not be possible to construct an enough robust and reliable ECG beat segmentation, which supports multiple beat types.

In our system a beat classifier unit supports the required a priori information of the beat type. The segmentation can be carried out based on this information, using beat-specific physiological-medical background knowledge as well (e.g. pathophysiological range of various time intervals inside a given type of beat). This solution increases the specificity and selectivity of the ECG beat segmentation method. Due to this considerations, we realized separated beat segmenters for each supported 
beat types, currently normal sinus rhythms (NB), premature ventricular contractions (PVC) and aberrated atrial premature contractions (APC). The beat segmenter units use the linearized version of the time-domain ECG signal, using time-domain measures and metrics in the same manner as a human cardiologists does it. During the design of the system we considered its feasibility and viability in real applications. Although the current prototype version of the system is implemented in Matlab, we designed the system to be portable for practical implementations. The modularity and scalability of the system allows the flexible incorporation of further beat segmenter units for other beat types.

\section{Methods}

Overview

The structure of the proposed hybrid ECG classifier and segmenter system is illustrated in Fig. 2. The signal flow is represented by numbered arrows, which will be referred during the description in brackets.

The sampled and digitized ECG signal enters the system on the left side of the figure. The ECG signal is first buffered (1) and then filtered due to the unwanted signal spikes and noises. The filtered ECG signal (2) is fed into the R-peak detector and into the beat isolator. The R-peak detector obtains the position of the R-peaks in the filtered ECG signal. The beat isolator uses this R-peak information (3) and the filtered ECG signal (2) to isolate the subsequent ECG beats from each other.

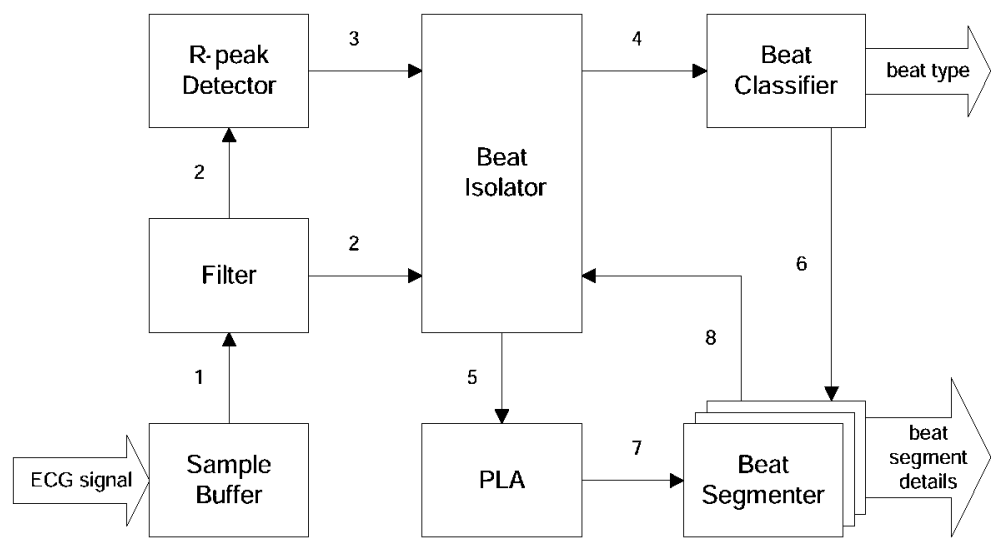

Fig. 2. The structure of the hybrid ECG segmenter. The numbered arrows symbolize the signal flow in the system. For their meaning, please refer to the text 
Once the beats are isolated, are first classified. This is the task of the beat classifier, which determines the type of the ECG beat using a normalized version of the beat pattern (4). Using the determined beat type, the beat classifier selects the corresponding beat segmenter (6). On the other hand, the samples of the isolated beat (5) are fed to the PLA module, which linearly approximates the ECG beat (7). The beat segmenter selected by the beat classifier implements the morphological analysis and segmentation of the isolated and linearly approximated beat. A feedback signal (8) is provided from the beat segmenters to the beat isolator for the adaptive adjustment of the isolation. The outputs of the system are the beat type and the beat segment details.

The following part of this section will handle each module of the illustrated system detailed.

\section{Sample buffer}

The goal of the proposed system is to provide real-time and on-line ECG segmentation with a maximal time delay of one heart beat. The sample buffer stage in front of the filter unit receives 128 samples independently of the used sampling rate. The sampling rate can be selected in the $250 \mathrm{~Hz}-1 \mathrm{kHz}$ range, and this selection affects only the timing constants of the beat segmenters described later in this paper, but not the sample buffer size.

If the buffer gets full, the buffered samples are passed to the filter stage and the buffer will be emptied and reset.

\section{Filter}

The filter stage provides the elimination of unwanted ECG signal components. These signal components can be high frequency (e.g. muscle noise, power line noise, electromagnetic interference) and low frequency (e.g. base line wandering, effect of respiration) disturbances. The filtering is carried out on the last 128 buffered samples (1).

The literature provides a number of useful methods for the ECG filtering. Many of them are based either on digital filter banks (11) or wavelet transform (12). Due to our previous researches in the field of ECG filtering, we chosen the last solution. 
The Wavelet Transform (WT) is widely used in engineering and usually devoted to the time frequency analysis of non-stationary signals, allowing to study the temporal evolution of the signal's spectra. The WT of a time-continuous signal $f(x)$ is defined by the following convolution integral:

$$
W_{s} f(x)=f(x)^{*} \Psi_{s}(x)=(1 / s) \int f(t) \Psi((x-t) / s) d t
$$

where $s$ is a scale factor, and $\Psi_{s}(x)=1 / s \Psi(x)$ is a scaled version of the original mother wavelet $\Psi(x)$. Calculating the WT at lower scales allows the study of the low frequency properties of the original signal with a rude time resolution, while the higher scale transformations represent the high frequency components with a finer time resolution. Theoretically the scale factor can be an arbitrary value, but in the practical applications it is usually chosen to be always the integer factorial of 2 . This dyadic WT can be efficiently calculated for discrete time series $f(k)$, using the following formulas:

$$
\begin{aligned}
& A_{j} f(k)=\sum_{n \in Z} h_{n} A_{2 j-1} f\left(k-2^{j-1} n\right) \\
& D_{j} f(k)=\sum_{n \in Z} g_{n} A_{2 j-1} f\left(k-2^{j-1} n\right)
\end{aligned}
$$

where $A_{j}, j \in N$ is the filtered (approximated) signal component, where $A_{0} f(k)=f(k)$, and $D_{j}, j \in N$ is the detail component on the $j$ th dyadic scale. The coefficients $h_{n}$ and $g_{n}$ are coefficients of an equivalent low pass filter $H$ and a high pass filter $G$, respectively. The formulas produce the wavelet decomposition of the signal at the $j$ th dyadic scale. In our case only one previously selected $A_{j} f(k)$ is to be calculated, so only the coefficients $h_{n}$ are relevant. We chosen the coif 2 wavelet as mother wavelet, which is a member of the Coiflet wavelet family.

The used filter coefficients were $(n=12)$ :

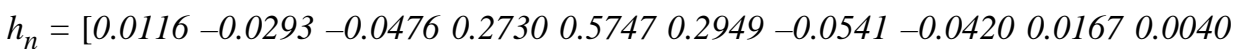
$-0.0013-0.0005]$

We used the third dyadic scale for the purpose of ECG filtering. This scale has been proven to be good for noise reduction without significantly affecting the signal's original shape. The calculation of $A_{3} f(k)$ can be carried out using the formula of $A_{j} f(k)$ above with triple recursion. 
Detecting the position of the R-peaks in the filtered ECG signal (2) is a wellknown problem. Many solutions are provided for this purpose (13). Some of them are very robust and highly reliable. In our case we applied a fast and - in case of an enough good filtered signal - quite robust method. This method combines a very simple adaptive threshold value monitoring with the consideration of the difference plot (first derivative of signal). An R-peak is detected if the signal reaches a desired threshold, which is adaptively adjusted using the previous R-peak values. Then the detection is verified on the first derivative of the signal, i.e. the first derivative should change its sign around the R-peak. The output of the detector (3) $r$ is an $n$-dimensional vector, where $n$ is the dimension of the input signal (2), and $r(i)=1$ if an R-peak has been detected at the $i$ th position and $r(i)=0$ in other cases.

\section{Beat isolator}

The beat isolator receives and merges the filtered signal parts (2), isolates the individual ECG beats (5) using the R-peak information (3), and produces a normalized beat pattern (4) for the beat classifier. The isolator works with one beat delay, i.e. the temporal place of the last recognized and isolated beat succeeds the last detected Rpeak. In other words: always the last complete ECG beat is isolated.

The last two R-peak positions and the end position of the last recognized ECG segment are considered for the isolation. If two consecutive R-peaks would not be yet available, then the beat isolator temporary buffers the signal fragments received from the filter. The isolation starts only if two consequetive R-peaks are available. The end position of the last recognized ECG segment (8) is returned by the beat segmenter and it marks the start position of the new beat. At the first beat or if the beat segmenter fails and cannot recognize any segment in the last ECG beat then - as a rule-of-thumb - the starting point will be set $300 \mathrm{~ms}\left(P_{d e f}\right)$ before the subsequent R-peak. The end position of the beat is determined by the second R-peak, and considered to be $200 \mathrm{~ms}$ before this. (The average of P-wave length is $100-300 \mathrm{~ms}$ in normal cases.)

Figure 3 illustrates this method, where the long-dashed lines mark the detected R-peaks and the filled dots on the ECG signal symbolize the end points of the last recognized ECG segment (8) (T-wave). Please note that the start of the first beat is determined by using the $P_{d e f}$ value mentioned above. 


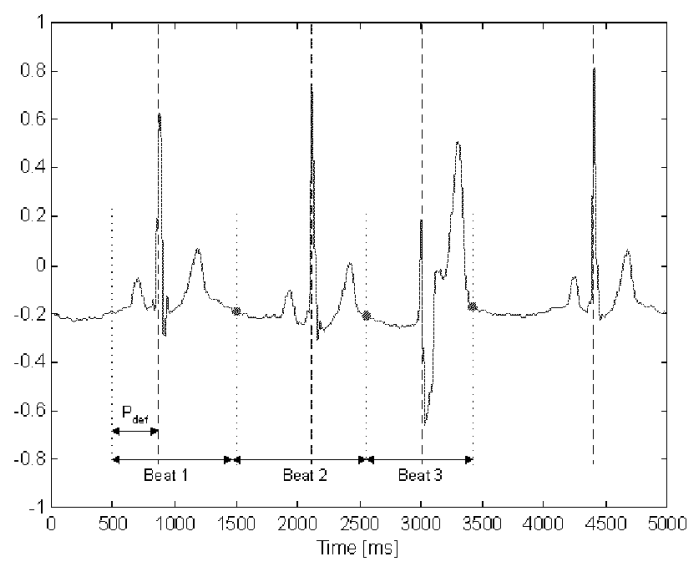

Fig. 3. The working principle of the beat isolator

\section{Beat classifier}

The task of the beat classifier is to determine the type of the actual beat and then select the corresponding ECG segmenter (6). The classifier is based on a neural network. Neural networks were applied successfully in the field of ECG beat classification $(14,15,16)$. We used a feed-forward neural network to classify the beat pattern. The input layer of the network receives a normalized version of the isolated time-domain beat pattern (4) consisting of only 80 sample points, which is about the one-fifth of the original sample number. We considered this number due to performance considerations, resulting in a neural network with 80 input units. The normalization takes place in the beat isolator, which four times upsample the original beat pattern and then equidistantly resample the 80 points. The baseline and the magnitude of the signal is normalized as well.

Regarding this 80 points resolution it is obvious that the diagnostic meaning of the signal (i.e. exact length, elevation, shape and area of the individual ECG waves) can be lost, but it is still appropriate for the purpose of beat classification.

We defined two hidden layers with 32 and 8 neurons, respectively. The output layer consists of 2 neurons, encoding currently 4 possible beat classes: NB, PVC, APC and unknown $(\mathrm{X})$. The network was trained using the resilient backpropagation (rprop) learning algorithm. We used 32 training patterns for each beat types. The neurons of the hidden and output layers have the traditional sigmoid as activation function. Figure 4 shows the topology of the resulted neural network of the beat classifier. 


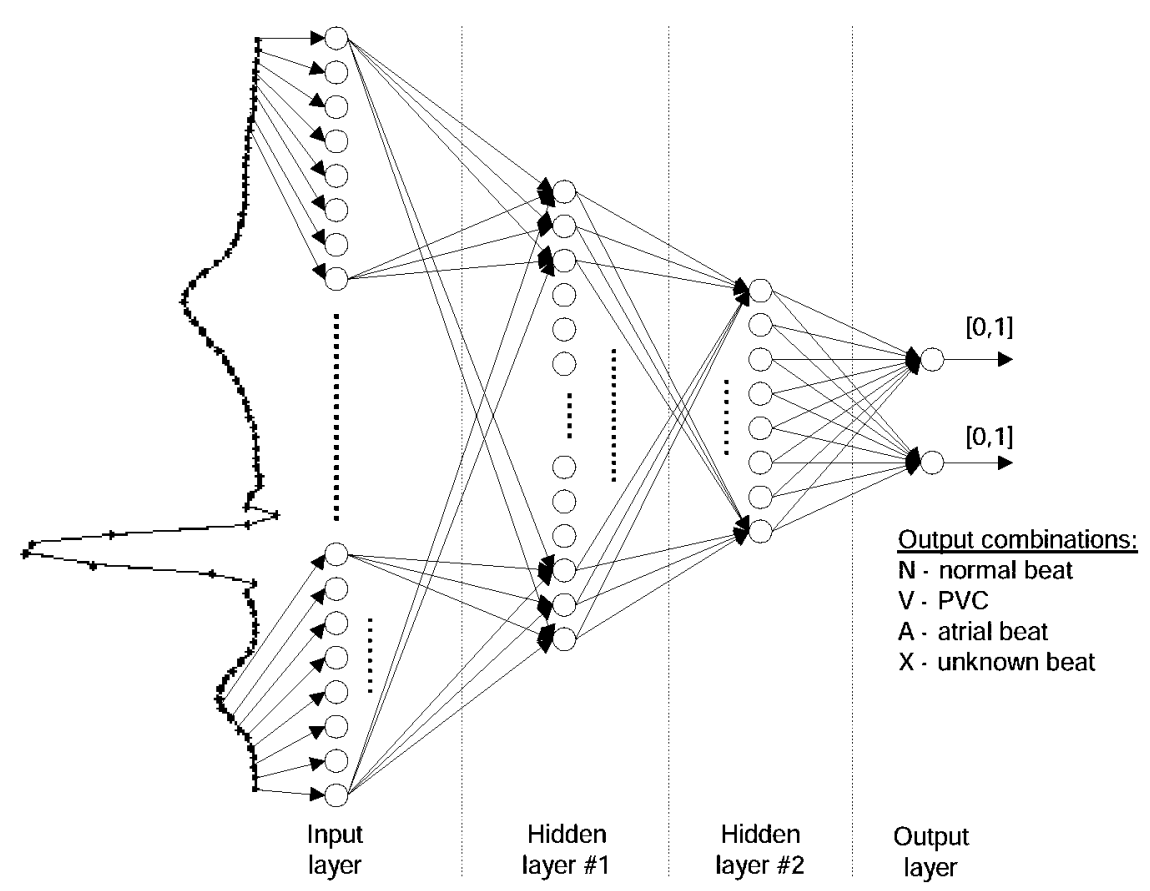

Fig. 4. The feed forward neural network of the beat classifier

Although the current version of the ECG segmenter system is running on a desktop PC, we considered the computational performance of the system during the design. This led us at selecting the number of the input units.

\section{The PLA}

This unit is responsible for the piecewise linear approximation (PLA) of the isolated beat image (5). The PLA algorithm applied in this paper is a modified version of the PLA used in Vullings et al. (4).

The idea of using PLA for the purposes of ECG beat segmentation originates in the fact, that PLA allows the time-domain analysis and segmentation of the ECG. Thus traditional cardiology metrics can be later incorporated and applied during the segmentation of the signal. 
The filtered and isolated ECG beat is regarded as a vector $y=[y(1) y(2) \ldots y(k)]$, consisting of $k$ sample points, where $y(i)$ is the sampled amplitude of the ECG beat at the given discrete time index $(0<i \leq k)$. The PLA version of this vector (7) is a set of straight line sections, where each linear section is defined by the pair $(d, m)$, where $d$ is the length and $m$ is the slope of the linear section, respectively. Thus the linear approximated ordinates of the section between the start and end abscissas $\left(x_{s}<x_{k} \leq x_{e}\right)$ is given by:

$$
\hat{y}_{k}=s\left(x_{k}-x_{s}\right)+\hat{y}_{s}
$$

where $y_{s}$ is the ordinate at the start point, $s$ and $e(0<s<e)$ are the indexes of the start end points of the section, respectively.

The length and the slope of the section are given by:

$$
\begin{gathered}
d=x_{e}-x_{s} \\
m=\frac{\hat{y}_{e}-\hat{y}_{s}}{d}
\end{gathered}
$$

At the calculation of the set of linear sections $(d, m)$ we considered the following error metrics:

$$
\varepsilon=\frac{\sum_{k=s}^{e}\left|\hat{y}_{k}-y_{k}\right|}{m^{2}+1}
$$

The goal of the PLA is to produce as few linear sections as possible. Of course the less the number of sections are the bigger the approximation error $\varepsilon$ can be. The PLA algorithm uses $\varepsilon$ to determine the length of the section. If the current approximation error remains below a desired maximal value $\left(\varepsilon<\varepsilon_{\max }\right)$, then the length of the section can be enlarged. $\varepsilon_{\max }$ means an upper limitation for the PLA. To avoid the too low fragmentation, we considered $l_{\min }$, too. $l_{\min }$ is the minimal length of a section, and the minimal step of section enlargement (i.e. each linear section approximates $i \cdot l_{\text {min }}$ sample points in the original signal, where $i>0$ is an integer number). 
The PLA applied in our system is shown in Listing 1 . We used the same notations as above.

Listing 1

The PLA algorithm

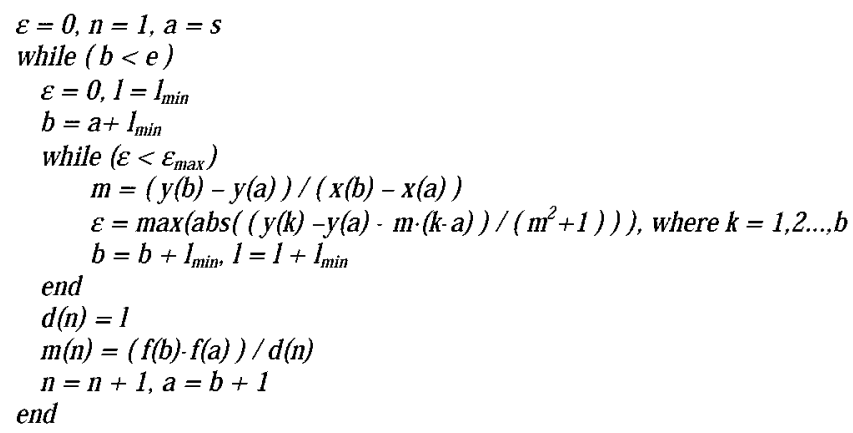

The presented algorithm produces a PLA of signal $y$ on the interval $(s, e)$, using $l_{\text {min }}$ and $\varepsilon_{\text {max }}$. The algorithm results in $n$ piece of $(d, m)$ pairs, arranged in vectors $d$ and $m$, respectively.

For the ECG signals we used, the values $l_{\text {min }}=4$ and $\varepsilon_{\text {max }}=0.01$ were chosen. These selections allowed the appropriate fine approximation of the original ECG signal in considerable processing time.

\section{Beat segmenters}

The task of the beat segmentation is to deliver the various measures and metrics of the ECG beat (e.g.: length and height of individual ECG-waves, length of specific time intervals).

Since the segmentation will take place in time domain (i.e. on the PLA produced linear sections (7) with given lengths and slopes), it was considerable to apply a method that uses time-domain metrics of the signal. This approach is similar that of a human cardiologists.

We designed a fast and flexible state-driven method to analyze and segment the ECG beats. Thus each beat segmenter is based on a finite state machine. The states represent segmentation or checking phases (e.g.: $\mathrm{P}$-wave rising, $\mathrm{T}$-wave falling, check of P-wave length, check of ST-interval). The transition between the phases happens on the change of some signal properties, using a priori physiological information. 
The following metrics are used during the segmentation, whereby threshold values are examined (Fig. 5):

- $\quad$ slope ranges

- length of an ECG-wave

- length of an interval

- positive or negative peak amplitude of an ECG-wave

- $\quad$ energy of an ECG-wave

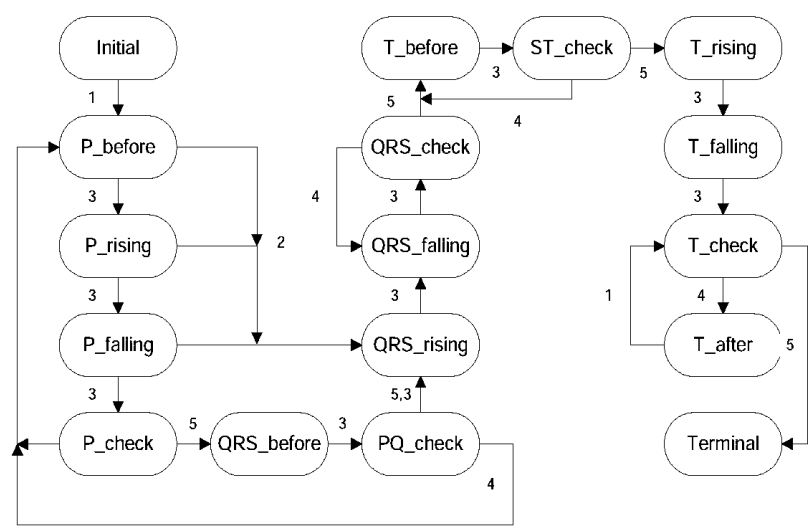

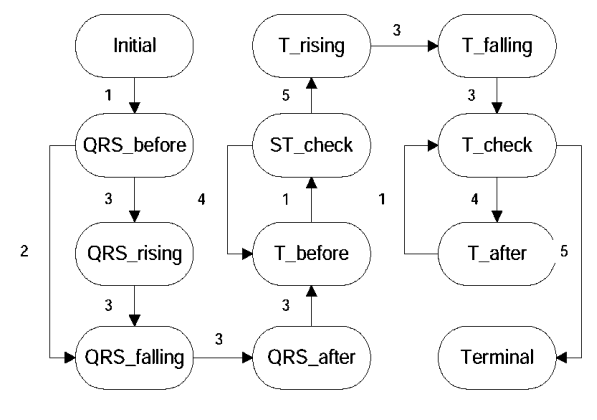

(b)

(a)

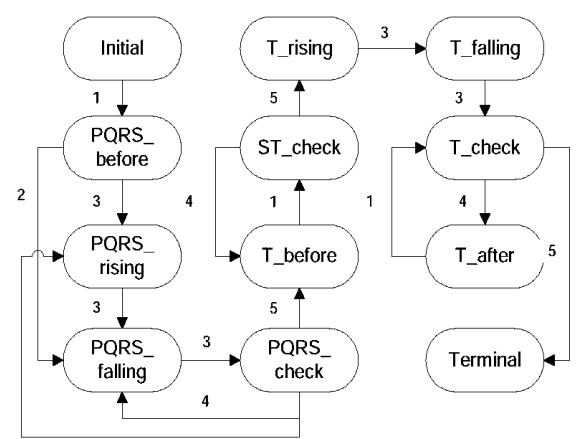

(c)

Fig. 5. The state machines of the beat segmenters: (a) - NB, (b) - PVC, (c) - APC. The individual segmentation states are displayed in rounded boxes, the state transitions are marked by numbered arrows. The cause of transitions: 1 - unconditional transition, 2 - R-peak is reached, 3 - slope is in expected range, 4 - length of interval is not as expected, 5 - length of interval is as expected 
The first three metrics are provided by the PLA, the latter two require additional calculations.

As it is now clear, the PLA supports the $n$-dimensional vectors $d$ and $m$. Let's suppose that an ECG-wave is approximated by the sections having the indices: $s, s+1$, $s+2, \ldots, e$, where $0<s<e \leq n$. In this case we defined the positive and negative peaks of the ECG-wave:

$$
\begin{aligned}
& h_{+}=\max \{m(k) \cdot d(k)\} \\
& h_{-}=\min \{m(k) \cdot d(k)\}
\end{aligned}
$$

where $s \leq k \leq e$.

The energy of an ECG-wave is approximated by the term:

$$
E=\sum_{k=s}^{e}|m(k) \cdot d(k)| \cdot d(k)=\sum_{k=s}^{e}|m(k)| d^{2}(k)
$$

The segmenter to be used at the actual ECG beat is selected by the beat type (6), provided by the beat classifier. Currently we realized three beat segmenters for NB, PVC and APC beats. If the selected beat segmenter succeeds, it returns the end position of the last detected ECG segment (8) to the beat isolator.

Table I contains the most interesting features of the various beat types from the cardiologist's point of view.

Table I

Segment details returned by the individual beat segmenters

\begin{tabular}{|l|l|l|}
\hline \multicolumn{1}{|c|}{ NB } & \multicolumn{1}{|c|}{ PVC } & \multicolumn{1}{c|}{ APC } \\
\hline P-wave length, height and area & QRS-length, height, area & PQRS- length, height, area \\
PQ interval & ST interval & ST interval \\
QRS- complex length, height and area & T-wave length, height, area & T-wave length, height, area \\
ST interval & QT interval & QT interval \\
T-wave length, height and area & RR interval & RR interval \\
QT interval & & \\
RR interval & & \\
\hline
\end{tabular}

The developed segmenters deliver all these measures. Figure 5 illustrates the finite state machines of the individual beat segmenter units. The numbers on the arrow symbolize the cause of the various state transitions. 
Table II

300 beat excerpts of these signal records were used

\begin{tabular}{|c|c|c|c|c|c|c|c|c|c|}
\hline $\begin{array}{l}\text { Rec. } \\
\text { No. }\end{array}$ & $\begin{array}{l}\text { Record name, } \\
\text { Database }\end{array}$ & $\begin{array}{l}\text { Beat } \\
\text { types }\end{array}$ & Rhythms & $\begin{array}{l}\text { Beat } \\
\text { annot. }\end{array}$ & $\begin{array}{l}\text { Wave } \\
\text { annot. }\end{array}$ & $\mathrm{S} / \mathrm{N}$ & $\mathrm{P}$ & $\begin{array}{l}\text { QR } \\
\text { S }\end{array}$ & $\mathbf{T}$ \\
\hline R1 & Sel103, QTDB & $\begin{array}{l}\mathrm{NB} \\
\mathrm{APC}\end{array}$ & $\begin{array}{l}\text { normal sinus, } \\
\text { supraventricular } \\
\text { ectopy }\end{array}$ & $\mathrm{Y}$ & $\mathrm{Y}$ & G & $\mathrm{S}$ & G & G \\
\hline R2 & Sel116, QTDB & $\begin{array}{l}\text { NB, } \\
\text { PVC }\end{array}$ & $\begin{array}{l}\text { normal sinus, } \\
\text { ventricular ectopy }\end{array}$ & $\mathrm{Y}$ & $\mathrm{Y}$ & S & S & G & G \\
\hline R3 & Sel117, QTDB & NB & normal sinus & $\mathrm{Y}$ & $\mathrm{Y}$ & $\mathrm{S}$ & G & G & G \\
\hline R4 & Sel123, QTDB & $\begin{array}{l}\text { NB, } \\
\text { PVC }\end{array}$ & ventricular ectopy & $\mathrm{Y}$ & $\mathrm{Y}$ & S & G & S & G \\
\hline R5 & $\begin{array}{l}\text { Sel16265, } \\
\text { QTDB }\end{array}$ & NB & normal sinus & $\mathrm{Y}$ & $\mathrm{Y}$ & $G$ & $\mathrm{~S}$ & G & G \\
\hline R6 & $\begin{array}{l}\text { Sel16272, } \\
\text { QTDB }\end{array}$ & NB & normal sinus & $\mathrm{Y}$ & $\mathrm{Y}$ & G & G & G & G \\
\hline R7 & 201, MITDB & $\begin{array}{l}\text { NB, } \\
\text { PVC, } \\
\text { APC }\end{array}$ & $\begin{array}{l}\text { normal sinus, } \\
\text { atrial fibrillation, } \\
\text { ventr. ectopy }\end{array}$ & $\mathrm{Y}$ & N/A & S & $\mathrm{S}$ & S & S \\
\hline R8 & 202, MITDB & $\begin{array}{l}\text { NB, } \\
\text { PVC, } \\
\text { APC }\end{array}$ & $\begin{array}{l}\text { normal sinus, } \\
\text { atrial fibrillation, } \\
\text { ventr. ectopy }\end{array}$ & $\mathrm{Y}$ & N/A & $S$ & $P$ & G & G \\
\hline $\mathrm{R} 9$ & Slp03, SLPDB & NB & normal sinus & $\mathrm{Y}$ & N/A & $\mathrm{S}$ & G & G & $\mathrm{P}$ \\
\hline R10 & Slp59, SLPDB & NB & normal sinus & $\mathrm{Y}$ & N/A & $\mathrm{P}$ & $\mathrm{P}$ & G & G \\
\hline
\end{tabular}

Legend: $\mathrm{S} / \mathrm{N}=$ signal/ noise ratio, $\mathrm{P}, \mathrm{QRS}$ and $\mathrm{T}$ : quality of $\mathrm{P}$-waves, QRS-complexes and T-waves in the record, respectively: $\mathrm{P}$ - poor, $\mathrm{S}$ - sufficient, $\mathrm{G}-$ good.

\section{Signal records}

The ECG signal records used in this study were provided by, and are available without cost from, PhysioNet, a public service of the Research Resource for Complex Physiologic Signals. We used the annotated records of the MIT-BIH QT Database (18), the MIT-BIH Arrhythmia Database and the MIT-BIH Polysomnographic(17). We selected the first ECG-channel in case of the two-channel ECG records of the QT Database and Arrhythmia Database. At the polysomnographic records contained only 
single-channel ECG signals. The records were taken from different patients. Table II contains the details of the applied signal records. We selected 10 records, and used continuous excerpts of 300 beats from each of the records. Thus the total number of beats was 3000 .

\section{Results}

The first version of the system was implemented in Matlab. The beat classifier and the beat segmenter units play the most important role in the system, thus we emphasize the results of these modules.

\section{Beat classifier}

For the training of the neural network in the beat classifier we selected 32 beat patterns of each type NB, PVC and APC from different records randomly. The network was trained with the rprop algorithm in 1000 epochs (summed mean square error, $S S E<0.025)$. We used the $S P$ specificity and the $S E$ selectivity at the evaluation of the beat classifier. We defined:

$$
S P=n_{+x} / N_{x} \text { and } S E=1-\left(N_{x}-n_{+x}\right) /\left(N-N_{x}\right),
$$

where $n_{+x}$ is the number of correctly classified beats of type $x, N_{x}$ is total number of beats of type $x$, and $N$ is the total number of all beats. In our record excerpts $N=3000$, $N_{N B}=2680, N_{P V C}=252$ and $N_{A P C}=68$. Table III contains the test results of the beat classifier.

\section{Table III}

Test results of the beat classifier for all 3000 test beats

\begin{tabular}{|l|c|c|}
\hline Beat Class & SP $(\%)$ & SE $(\%)$ \\
\hline NB & 92.5 & 97.3 \\
\hline PVC & 97.8 & 96.4 \\
\hline APC & 94.1 & 89.3 \\
\hline Overall & 94.8 & 94.3 \\
\hline
\end{tabular}




\section{Beat segmenters}

For the validation of the ECG segmentation, the records R1-R6 contain special wave annotations consisting of the onset and offset positions of the individual waves. Within each record, only about 30-40 representative beats were manually annotated by cardiologists, and the remaining beats were annotated by a computerized algorithm, using a differential threshold detection algorithm (6). Due to the imprecise computer generated annotations, especially at the poor quality P-waves, at the onset and offset of the QRS-complexes and in case of not normal sinus rhythms, we discarded them, and used only the cardiologist's annotations. In case of the other beats and at records R7R10 we asked a cardiologist to give us guidelines for the evaluation, and we considered Braunwald E.: Heart Disease (1), too. The assessment of the segmentation was a semiautomatic process, supported by a Matlab routine, which display a comprehensive information window (Fig. 6). The window is divided into 4 subplots, displaying various state of the signal processing chain.

During the assessment we regarded distinguishly the isolation of the P-wave, of the QRS-complex and of the T-wave. We considered the isolation as correct if it corresponded either to the manually annotated or to visually verified segment borders with at least $90 \%$ precision. Table IV shows the success rate of the segmentation in case of the individual ECG segments (P, QRS and T) and for the whole beats, too.

\section{Discussion}

We used signals from ten records of three different ECG reference signal databases, originating from different subjects. At the test and evaluation we considered 3000 ECG beats, consisting also noisy and rather unclear signal segments. The current beat classifier recognized the NB, PVC and APC beats at more than $94 \%$ average selectivity and specificity. The segmenters could properly segment the $95 \%$ of the specific classified beats. Thus we found the overall signal performance (regarding the full signal processing chain) higher than $89 \%$. This result is quite considerable, regarding that the applied signals contained noisy segments and unknown beat types of up to $8 \%$. If we do not consider these signal segments, the overall system performance is above $93 \%$. 

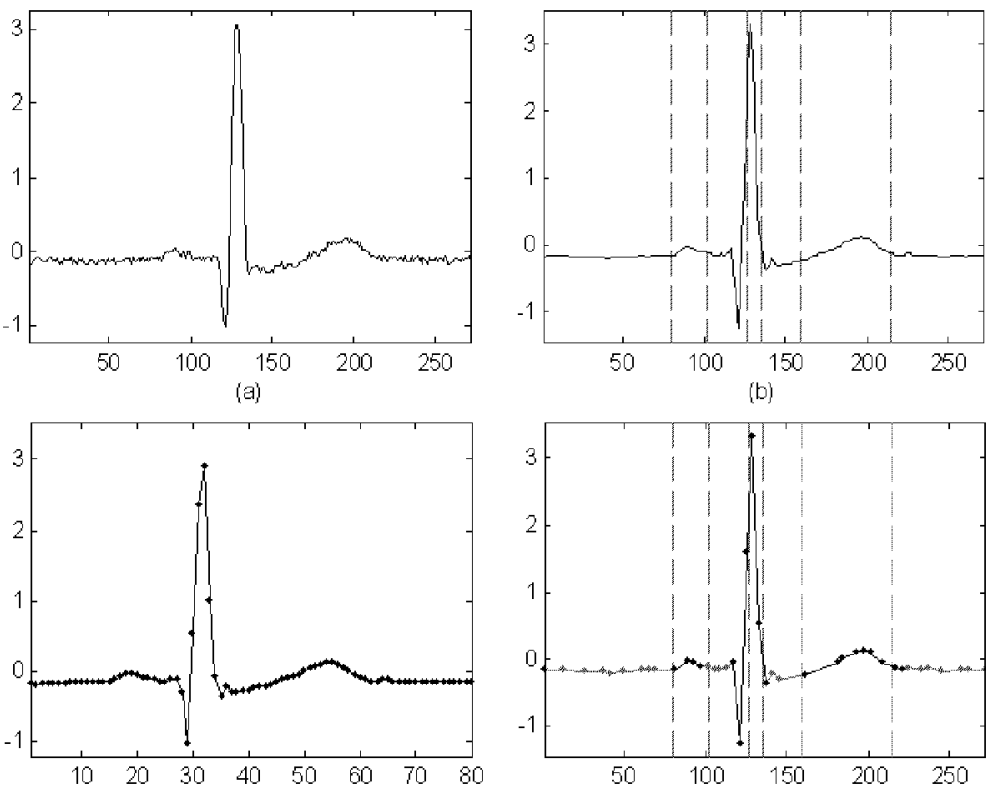

Beat class: NB

$\mathrm{P}$ length $=82 \mathrm{~ms}, \mathrm{P}$ height $=0.18, \mathrm{P}$ area $=11.5$

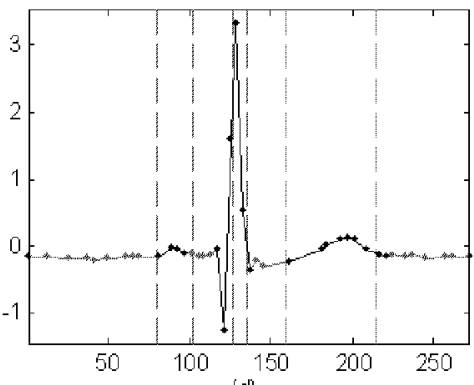

Sampling rate: $250 \mathrm{~Hz}$

QRS_length $=104 \mathrm{~ms}, \mathrm{QRS}$ _height $=3.02, \mathrm{QRS}$ _area $=127.4$

T length $=207 \mathrm{~ms}, T$ height $=0.39, \mathrm{~T}$ area $=55.3$

$\mathrm{PQ}=87 \mathrm{~ms}, \mathrm{ST}=91 \mathrm{~ms}, \mathrm{QT}=402 \mathrm{~ms}$

Fig. 6. The evaluation of segmentation. (a) - original ECG beat, (b) - Filtered signal, (c) - Normalized beat for beat classification, (d) - the PLA's and segmented beat. The segmented P, QRS and T waves are highlighted on subplot (d). The dashed vertical lines showing the segment borders of the human cardiologist's. The detailed values calculated by Matlab are displayed in the bottom part of the window 
Table IV

Test results of the beat segmenters

\begin{tabular}{|c|c|c|c|c|}
\hline \multirow{2}{*}{ Rec. No. } & \multicolumn{5}{|c|}{ P } & \multicolumn{2}{|c|}{ QRS } & Whole beat \\
\cline { 2 - 5 } R1 & 93.6 & 98.3 & 95.4 & 95.8 \\
\hline R2 & 96.7 & 98.8 & 96.9 & 97.5 \\
\hline R3 & 97.1 & 98.5 & 87.7 & 94.4 \\
\hline R4 & 95.7 & 90.3 & 93.3 & 93.1 \\
\hline R5 & 97.3 & 99.3 & 99.1 & 98.6 \\
\hline R6 & 94.5 & 98.7 & 88.3 & 93.8 \\
\hline R7 & 92.1 & 95.3 & 97.2 & 94.9 \\
\hline R8 & 95.2 & 95.1 & 96.6 & 95.6 \\
\hline R9 & 96.9 & 93.3 & 84.1 & 91.4 \\
\hline R10 & 93.2 & 98.5 & 93.5 & 95.1 \\
\hline & & & & 95.02 \\
\hline
\end{tabular}

Figure 7 shows the segmentation result of four different ECG beats. The filtered ECG signals can be seen in the top row of the figure. The corresponding segmentation results are displayed in the underlying plots of the bottom row. The recognized Pwaves, QRS-complexes and T-waves are marked with darker line segments. The individual time interval details are not displayed here due to space considerations. Please note that Figures 7a, 7b and 7c show various type of ECG beats from record R8, and Figure $7 \mathrm{~d}$ is a normal sinus rhythm of another subject from record R6 using different sampling rate. Figure 7 consist unproblematic and nice signals. However these signals are most common in ECG records, real applications should handle noisy signals, too. Thus selected the applied test records to contain rather noisy signal sections as well.

Figure 8 shows the performance of the system in case of some problematic ECG signals. To demonstrate how effective the segmentation system is, we shunted the filter stage, so the beat classifier, the PLA and the beat segmenters used the unfiltered and noisy input signals. Figures $8 \mathrm{a}$ and $8 \mathrm{~b}$ consist signals with high frequency noise (muscle noise or patient movement) on the T-wave and on the P-wave, respectively. 


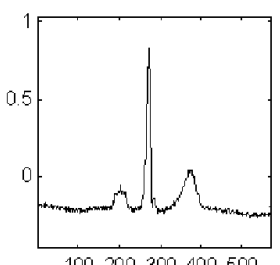

100200300400500

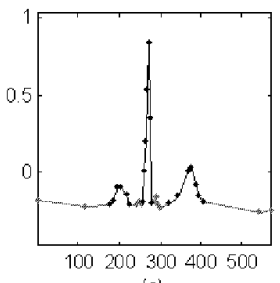

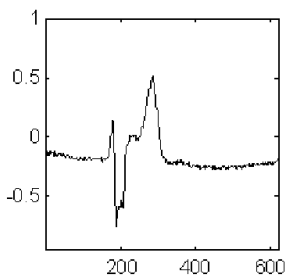
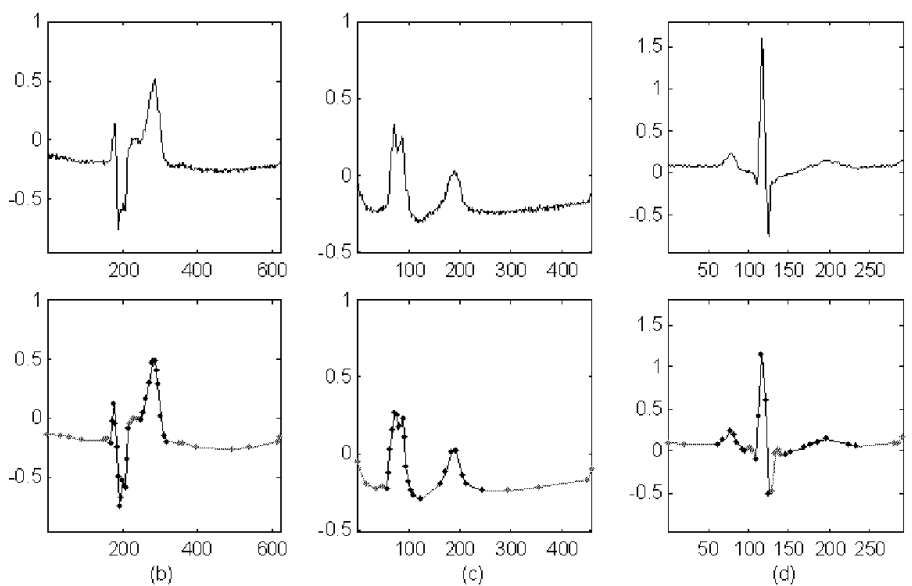

Fig. 7. Segmentation of various type of ECG beats: (a) - NB, (b) - PVC, (c) - APC, (d) - NB

Figures $8 \mathrm{c}$ and $8 \mathrm{~d}$ display signals with low frequency noise (baseline movement). As it can be seen in the figure, the segmentation system could recognize the individual ECG waves in case of rather noisy segments, too. The P-wave of Figure $8 \mathrm{~d}$ was not recognized, since it was very tiny and stayed below the desired threshold for the height and area of a P-wave. The good results in case of the high frequency noises (Figs 8a and 8b) can be explained with the low-pass filter characteristic of the applied PLA algorithm. Although the filter stage was shunted, the PLA could provide enough additional filtering to be able to remove the unwanted spikes. The low frequency noise (Figs 8c and 8d) was cancelled by the beat segmenter. The proper selection of the threshold values (i.e. signal slopes, individual interval lengths, wave areas) can provide additional resistance against the baseline drifts.

In spite of the very promising results we outline some bottlenecks of the system.

However the system was designed especially for the on-line analysis and segmentation of single-channel ECG records, we must note that one ECG channel cannot contain all the dynamics which would be required for a complex cardiac analysis. The single-channel ECG recording has its inherited problems of electrode displacement or loosing of contact, which can alter the measured waveform quite significantly. 

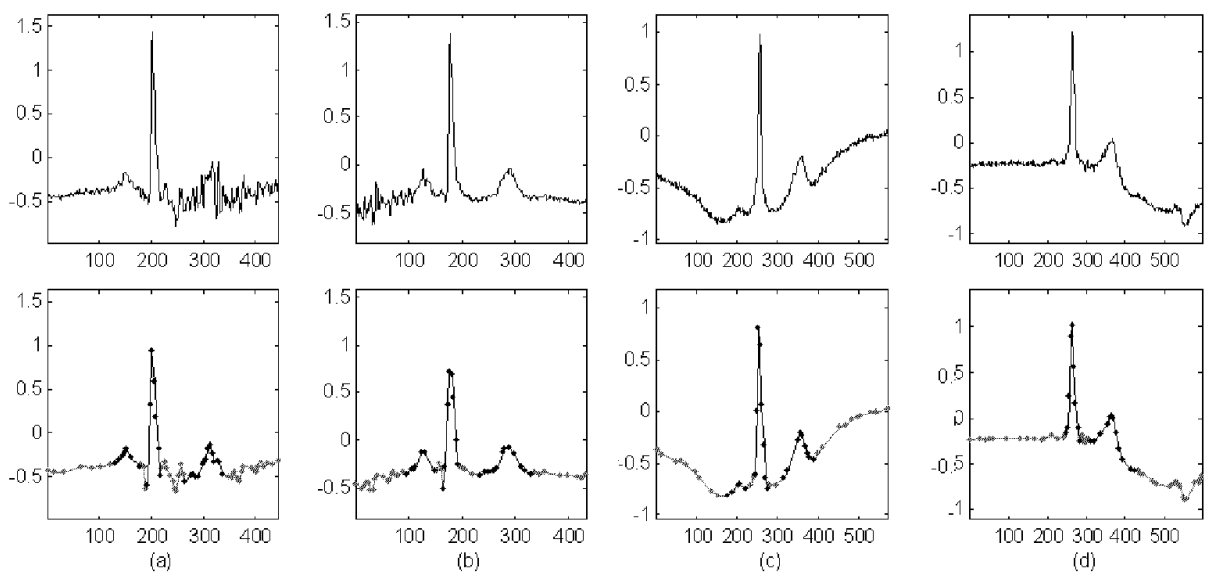

Fig. 8. Segmentation of unfiltered ECG signal. (a) (b) - noise, (c) (d) - baseline drift

The current system cannot segment the unknown beat types (classified as category $\mathrm{X}$ by the beat classifier). This problem can be solved by the training of other beat types and the incorporation of their corresponding beat segmenter units. However the number of the hidden units in the beat classifier's neural network should not increase significantly, due to performance considerations. On the other hand the false classified beats are segmented with an inappropriate segmenter, eventually resulting in faulty segments. Thus in case of practical applications the incorporation of a validation unit after the beat segmenter units is highly desirable. The validation unit should check the resulted segment details and should discard them in case of abnormal values.

Another problem is that the state transition threshold values used in the beat segmenters are currently patient-independent. We tried to find wide ranges for the individual thresholds values, which were properly worked in the selected ten signal records from ten different patients. However in real applications these threshold values should be selected patient specific, and they should be tuned adaptively.

Another challenging task would be to detect the $\mathrm{P}$ waves probably hidden in any fractions of the ECG. This would provide essential information in connection with certain arrhythmias. The increasing PQ distance might refer to Wenkebach-periodicity. The incorporation of such an analysis would require the alteration of the analysis window, since the current analysis window of one beat is not suitable for recognition of more complex events. 


\section{Conclusion}

The goal of the introduced system is to provide a robust, real-time and on-line beat segmentation using one-single ECG channel. The feasibility and viability of the system was verified by implementing a prototype in Matlab. The currently implemented system supports the segmentation of three different types of ECG beats (NB, PVC and APC), however other beat segmenters can be later easily integrated due to the modular system structure. The system requires moderate computational power, allowing the practical implementation in low-cost, stand-alone ECG monitors. The proposed new system structure resulted in robust and very promising segmentation performance.

As the further direction of research we are working on a patient adaptive threshold value tuner for the beat segmenter units and making studies on the detection of the P-waves buried in other fractions of the ECG.

\section{Acknowledgments}

This research and development project has been supported by the Hungarian National Research Fund grants No. OTKA T029830 and OTKA F029739.

\section{REFERENCES}

1. Braunwald, E.: Heart Disease. A Textbook of Cardiovascular Medicine. W. B. Saunders, Saint Louis 1997.

2. Fye, W. B.: Disorders of the heartbeat: A historical overview. Am J Cardiol. 72, 1055-1079 (1993).

3. Laguna, P., Jané, R., Caminal, P.: Automatic detection of wave boundaries in multilead ECG signals: Validation with the CSE database. Comput. Biomed. Research 27, 45-60 (1994).

4. Vullings, H. J., Verhaegen, M. H., Verbruggen, H. B.: ECG Segmentation Using Time-Warping. In: Advances in Intelligent Data Analysis, IDA-97, Springer Verlag, Berlin 1997, pp. 275-285.

5. Sedaaghi, M. H.: ECG Wave Detection Using Morphological Filters. Applied Sig. Proc. 5, 182-194 (1998).

6. Carrault, G., Senhadji, L., Bellanger, J., Le Pichon, J.P.: ECG Segmentation by Means of Change Detection Theory. Proc. Ann. Int. Conf. IEEE EMBS, Philadelphia 1990, pp. 817-818.

7. Sahambi, J. S., Tandon, S. N., Bhatt, R. P.: Using wavelet transform for ECG characterization. IEEE EMBS Magazine 16, 77-83 (1997).

8. Li, C., Zheng, C., Tai, C.: Detection of ECG Characteristic points using wavelet transforms. IEEE Trans. Biomed. Eng. 42, 21-28 (1995).

9. Kadambe, S., Murray, R., Boudreaux-Bartels, G. F.: Wavelet transform-based QRS complex detector. IEEE Trans. Biomed Eng. 46, 838-848 (1999).

10. Narayan, S. M., Smith, J.: Spectral analysis of periodic fluctuations in electrocardiographic repolarization. IEEE Trans. Biomed Eng. 46, 203-212 (1999).

11. Afonso, V. X., Tompkins, W. J., Nguyen, T. Q., Shen, L.: ECG beat detection using filter banks. IEEE Trans. Biomed Eng. 46 192-202 (1999). 
12. Szilágyi, L.: Wavelet-Transform-Based QRS Complex Detection in On-Line Holter Systems. Proc. Joint Conf. BMES-EMBS, Atlanta 1999, p. 271.

13. Friesen, G. M., Jannett, T. C., Jadallah, M. A., Yates, S. L., Quint, S. R., Nagle, H. T.: A comparison of the noise sensitivity of nine QRS detection algorithms. IEEE Trans. Biomed. Eng. 37, 85-98 (1990).

14. Ölmez, T., Dokur, Z., Yazgan, E.: Classification of ECG Waveforms Using a Novel Neural Network. Proc. Ann. Int. Conf. IEEE EMBS, Hong Kong 1998, pp. 1616-1619.

15. Maglevaras, N., Stamkopoulos, T., Pappas, C., Strintzis, M. G.: An adaptive backpropagation network for real-time ischemia episode detection. IEEE Trans. Biomed. Eng. 45, 805-813 (1998).

16. Xue, Q., Hu, Y. H., Tompkins, W. J.: Training of ECG signals in Neural Network Pattern Recognition. Proc. Ann. Int. Conf. IEEE EMBS, Philadelphia 1990, pp. 1465-1466.

17. Goldberger, A. L., Amaral, L. A. N., Glass, L., Hausdorff, J. M., Ivanov, P. C., Mark, R. G., Mietus, J. E., Moody, G. B., Peng, C. K., Stanley, H. E.: PhysioBank, PhysioToolkit, and Physionet: Components of a New Research Resource for Complex Physiologic Signals. Circulation 101, 215-220 (2000).

18. Laguna, P., Mark, R. G., Goldberger, A. L., Moody, G. B.: A database for evaluation of algorithms for measurement of QT and other waveform intervals in the ECG. Computers in Card. 24, 673-676 (1997). 